\title{
Sílice Mesoporosa como encapsulador de materiales de cambio de fase (PCM)
}

\section{Mesoporous silica as a phase change material encapsulator (PCM)}

\author{
GONZÁLEZ-CERVANTES, Nancy ${ }^{1} \dagger$, SALAZAR-HERNÁNDEZ, Mercedes ${ }^{2}$, CANO-LARA, \\ Miroslava $^{3}$ y SALAZAR-HERNÁNDEZ, Carmen ${ }^{1 *}$
}

${ }^{1}$ Unidad Profesional Interdisciplinaria de Ingeniería Campus Guanajuato, Instituto Politécnico Nacional, Av. Mineral de Valenciana No. 200 Col. Fracc. Industrial Puerto InteriorSilao de la Victoria, Guanajuato, México. C.P.36275

${ }^{2}$ Universidad de Guanajuato, Departamento de Ingeniería en Minas, Metalurgia y Geología, Ex Hacienda de San Matías S/N Colonia San Javier, Guanajuato, Gto, México. C.P.36020

${ }^{3}$ Instituto Tecnológico Superior de Irapuato, Departamento de Mecatrónica. Carretera Irapuato-Silao km 12.5 Colonia El Copal.

ID $1^{\text {er }}$ Autor: Nancy, González-Cervantes / ORC ID: 0000-0002-0494-9331

ID $1{ }^{\mathrm{er}}$ Coautor: Mercedes, Salazar-Hernández / ORC ID: 0000-0001-8039-8124

ID $2^{\text {do }}$ Coautor: Miroslava, Cano-Lara / ORC ID: 0000-0002-3335-2710

ID $3^{\text {er }}$ Coautor: Carmen, Salazar-Hernández / ORC ID: 0000-0002-6901-2937

DOI: $10.35429 / J T E N .2019 .9 .3 .14 .19$

Recibido 03 de Enero, 2019; Aceptado 30 Marzo, 2019

\section{Resumen}

Hoy en día el uso de fuentes de energía renovable efectivas son temas de investigación, siendo la radiación solar directa una de las mejores fuentes de energía. Sin embargo, el uso de esta forma de energía es optimizada con el desarrollo de tecnologías para su almacenamiento. Una de las técnicas propuestas para el almacenamiento de energía solar es la aplicación de materiales de cambio de fase (PCMs). Varios candidatos de materiales de cambio de fase como orgánicos e inorgánicos y sus mezclas han sido propuestos como almacenadores de energía ya que tienen un elevado calor latente. Sin embargo, una desventaja de estos materiales es su descomposición durante los ciclos de almacenamiento y liberación de energía, por lo cual, en este proyecto se propone encapsular los PCM en redes de sílice mesoporosa con la finalidad de incrementar la estabilidad térmica.

PCM, Sílice mesoporosa, Parafina, DSC

\begin{abstract}
Today, the effective renewable energy sources are research topics, with direct solar radiation being one of the best sources of energy. However, the use of this form of energy is optimized with the development of technologies for storage (TES). One of the proposed techniques for solar energy storage is the application of phase change materials (PCMs). Several candidates of phase change materials such as organic and inorganic and their mixtures have been proposed as energy storage because they have a high latent heat. However, a disadvantage of these materials is their decomposition during storage and energy release cycles, therefore, in this project it is proposed to encapsulate PCMs in mesoporous silica networks in order to increase thermal stability.
\end{abstract}

PCM, Mesoporous silica, Parafin, DSC

Citación: GONZÁLEZ-CERVANTES, Nancy, SALAZAR-HERNÁNDEZ, Mercedes, CANO-LARA, Miroslava y SALAZAR-HERNÁNDEZ, Carmen. Sílice Mesoporosa como encapsulador de materiales de cambio de fase (PCM). Revista de Ingeniería Tecnológica. 2019. 3-9: 14-19

\footnotetext{
* Correspondencia del Autor (Correo electrónico: msalazarh@ipn.mx)

$\dagger$ Investigador contribuyendo como primer autor.
} 


\section{Introducción}

Los materiales de cambio de fase (Phase Change Materials, PCM) son materiales que tienen un elevado calor latente (fusión o ebullición) o sensible; los cuales son empleados como almacenador de energía térmica [1]. Entonces, estos materiales han sido propuestos como nuevos sistemas de almacenamiento de energía (Thermal Energy Storage, TES) [2]. La Figura 1 muestra la clasificación de los TES indicando el tipo de materiales que han sido empleados hasta la fecha como PCM (materiales que emplean el calor latente como almacenador de energía). Los PCM pueden ser materiales orgánicos como: las parafinas, ácidos grasos, polímeros, azucares y mezclas eutécticas; en este caso se utiliza la entalpia latente de cambio de fase de sólido a líquido. Otro tipo de material como PCM son las sales inorgánicas y sales hidratadas, en estos materiales también utilizan el calor latente de fusión o cristalización como posible almacenador de energía [3].

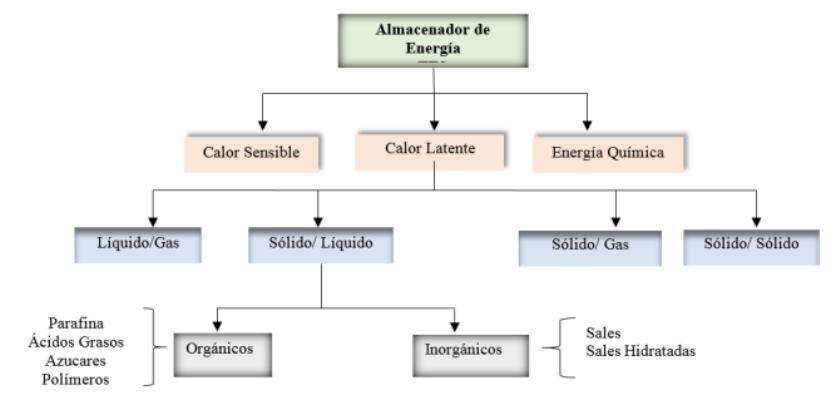

Figura 1 Clasificación de TES

Los PCM tienen como principal desventaja que a ciertos ciclos de uso presentan disminución en las capacidades de transferencia de energía térmica y de almacenamiento; lo anterior se debe principalmente a que durante la transformación de fases el material presenta cambios en el volumen molar [4]. Por lo que hoy en día se busca estabilizar los PCM encapsulándolos en sistemas estables que no disminuyan la capacidad de almacenamiento de energía pero estabilicen la estructura del material durante los procesos de transformación de fases. Entre los sistemas empleados como encapsuladores o estabilizadores de PCM se encuentra la sílice mesoporosa (MS, Mesoporous Silica) [5-6]. La MS es una red de sílice que en su interior contiene una gran cantidad de poros donde pueden alojar diferentes tipos de moléculas, entre las cuales se encuentran contaminantes como colorantes [7], plaguicidas [8], metales pesados [9], entre otros.
Entre los PCM que se han encapsulado en MS o nanopartículas de sílice (MSN, Mesoporous Silica Nanoparticle) se encuentran los ácidos grasos como son el ácido esteárico [10], ácido laurico [11], ácido adipico [12].

Los materiales de cambio de fase encapsulados en nanopartículas de sílice mesoporosa PCM/MSN son obtenidas principalmente a través de dos métodos de síntesis (véase Figura 2):

1) Síntesis Directa: La MSN es sintetizada a través de la metodología del tamiz molecular [4], donde se emplea una molécula de surfactante quien da forma a los poros de la sílice. En esta síntesis se adiciona el PCM de forma directa durante la formación y estructuración de la MSN (Figura 2, Esquema A).

2) Impregnación: En este método debe prepararse la MSN para impregnarla del PCM colocándola en contacto con una solución que contenga el PCM (Figura 2. Esquema B) o bien logrando que el PCM se adsorba en los poros de la sílice a través de aplicar presiones elevadas (Figura 2. Esquema C).

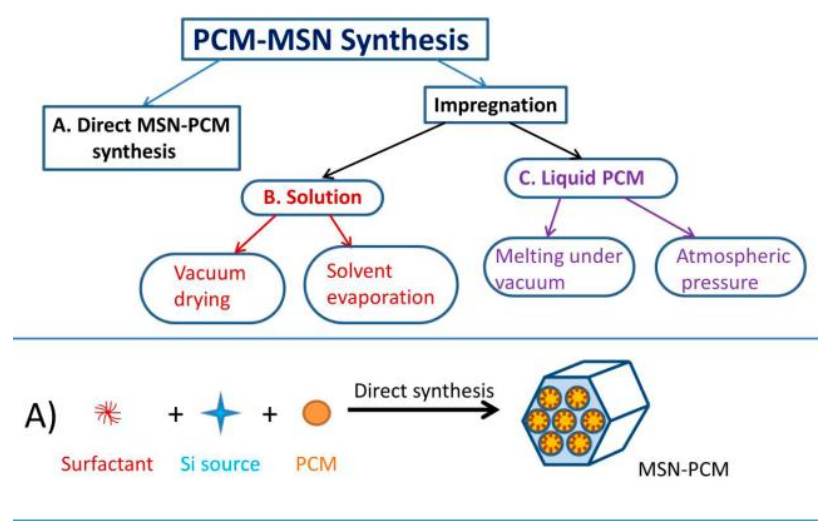

B)
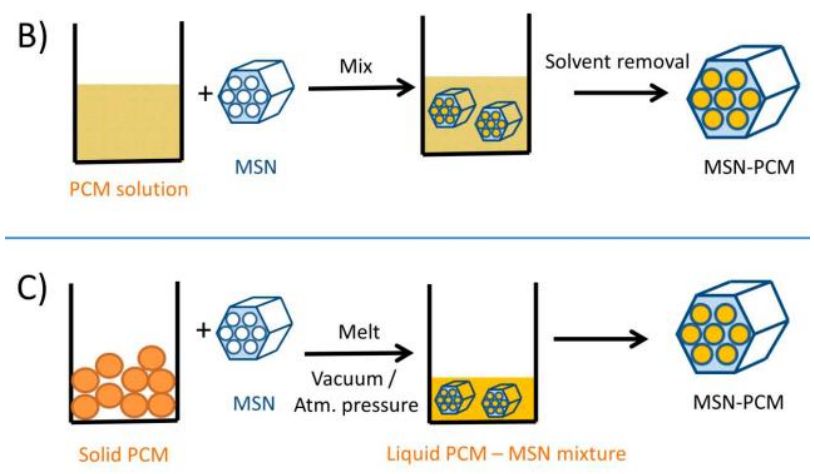

Figura 2 Métodos de obtención de PCM encapsulados en sílice mesoporosa [4] 
En este proyecto se propone explorar el encapsulamiento de un aceite vegetal en sílice mesoporosa (PCM/MSN) obtenidos con la metodología del tamiz molecular; se explorará el uso de pluronic como surfactantes que permita aglomerar el aceite para posteriormente depositar la sílice empleando tetraetilortosilicato como precursor. Los PCM/MSN se caracterizan por espectroscopia de infrarrojo para determinar la presencia del aceite dentro de la estructura de la sílice y por DSC para determinar la capacidad de almacenamiento del sistema.

\section{Metodología}

\section{Materiales}

Los PCM/MSN fueron sintetizados empleando tetraetil-orto-silicato (TEOS; 99\% Aldrich) como precursor formador de sílice, hidróxido de amonio (30\%, J.T. Baker) fue empleado como catalizador sol-gel, Pluronic F-127 (Aldrich) como surfactante y agua desionizada y etanol grado reactivo como disolventes. El PCM empelado fue aceite vegetal comercial.

\section{Síntesis de MSN}

En $300 \mathrm{~mL}$ de Etanol se disuelven $14 \mathrm{~g}$ de Pluronic F-127 ajustando el pH a 10 con $\mathrm{NH}_{4} \mathrm{OH}$. A esta disolución se adicionaron $20 \mathrm{~mL}$ de TEOS y $6 \mathrm{~mL}$ de agua desionizada. La disolución se mantiene por $24 \mathrm{hr}$ a temperatura ambiente y agitación moderada. El sólido formado se filtra y seca a $80^{\circ} \mathrm{C}$ por $4 \mathrm{hr}$. El pluronic es eliminado por extracción sólido/líquido colocando la MSN en reflujo por $24 \mathrm{hr}$ con Etanol a pH de 3. Finalmente el sólido se filtra y lava con etanol paras secarse a $80^{\circ} \mathrm{C}$ por $4 \mathrm{hr}$ y eliminar el remanente de pluronic por calcinanción a $400^{\circ} \mathrm{C}$ por $2 \mathrm{~h}$.

\section{Síntesis de PCM/MSN}

Los PCM/MSN fueron sintetizados por síntesis directa empleando la síntesis antes descrita para la MSN; se adicionaron diferentes cantidades de aceite que fueron de $1 \mathrm{~mL}, 2 \mathrm{~mL}$ y $3 \mathrm{~mL}$. El PCM fue adicionado a la disolución de Pluronic F-127 y la reacción se llevó acabo a $50^{\circ} \mathrm{C}$ para favorecer el encapsulamiento del aceite en el surfactante. Posteriormente se adicionó el TEOS para formar la MSN con el PCM adsorbido (PCM/MSN) y finalmente se llevó acabo la extracción del surfactante.

\section{Caracterización Química por Espectroscopía de Infrarrojo con Transformada de Fourier (IR-TF)}

El IR-TF permite identificar los principales grupos funcionales en la MSN y PCM/MSN determinando la composición de los materiales. Los espectros de IR se obtuvieron empleando un equipo IR-ATR-Nicolet iS-10 TA-Instrument, el barrido se realizó en una ventana espectral de 400 a $4000 \mathrm{~cm}^{-1}$.

\section{Isotermas de Adsorción-Desorción de $\mathbf{N}_{2}$}

Esta técnica de análisis permitió determinar las propiedades texturales (tamaño de poro, área superficial, volumen de poro) de la MSN y PCM/MSN. Las isotermas de adsorcióndesorción de $\mathrm{N} 2$ se obtuvieron a $77 \mathrm{~K}$ en un equipo Micromeritics ASAP-2010. Las muestras fueron desgasificadas a $70^{\circ} \mathrm{C}$ y $7 \square \mathrm{mHg}$. El área superficial se determinó por el método de Brunauer, Emmett y Teller (BET) y el diámetro promedio del poro por el método Barrett, Joyner y Halenda $(\mathrm{BJH})$ empleando la isoterma de desorción.

\section{Caracterización morfológica: Microscopía Electrónica de Barrido}

La microscopía electrónica de barrido permitió identificar la morfología obtenida para la MSN y los PCM/MSN. Las micrografías se obtuvieron con un equipo SEM-JOEL6510 plus.

\section{Caracterización Térmica}

El análisis térmico se realizó con un equipo TGA/DSC SDTQ600 TA-Instrument. El análisis termogravimétrico (TGA) fue realizado para cuantificar la cantidad de PCM adsorbida; dicho análisis se realizó bajo atmosfera oxidante en un rango de temperatura de 25 a $800^{\circ} \mathrm{C}$ con rampa de calentamiento de $10 \% \mathrm{~min}$.

\section{Análisis de esultados}

\section{Caracterización Química y Física}

La Figura 3a muestra los espectros de infrarrojo para la MSN donde se observan la formación de una superficie de sílice hidratada, agua adsorbida se observó con las señales a $3600 \mathrm{~cm}^{-}$ ${ }^{1}$ (v) y $1640 \mathrm{~cm}^{-1}(\delta)$. Además de identificarse los silanoles libres como una banda intensa a 945 $\mathrm{cm}^{-1}$. 
Por otra parte la red de sílice es identificada con los modos de vibración correspondientes a los siloxanos $\left(1150 \mathrm{~cm}^{-1}\right.$, vas y $570 \mathrm{~cm}^{-1}$, vs) [13]. La presencia de silanoles libres y agua fisisorbida permiten mejorar la capacidad de adsorción de la sílice con las moléculas orgánicas, como se muestra en la Figura 4 se incrementa las interacciones de tipo Van der Waals y puentes de hidrógeno.

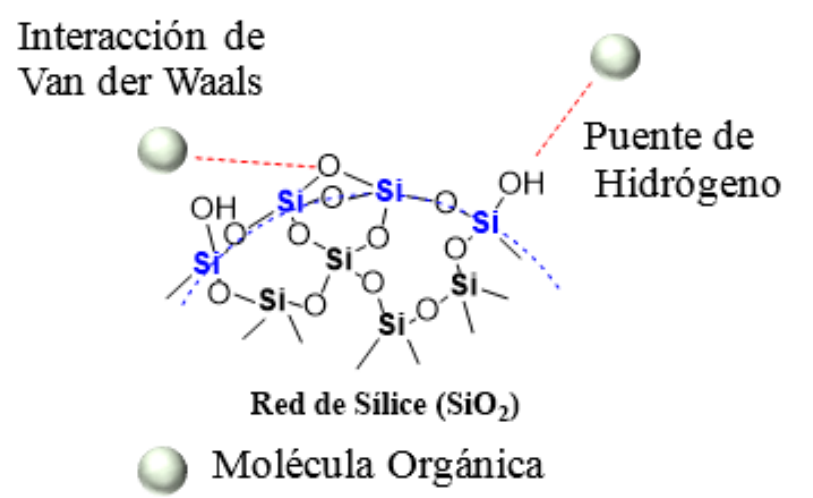

Figura 4 Efecto del agua en la estructura de la MSN sobre su capacidad de adsorción

En cuanto al PCM (Figura 3b) se observó una estructura correspondiente a un hidrocarburo, grupos - $\mathrm{CH}$ fueron observados de $2841 \mathrm{~cm}^{-1}$ a $3009 \mathrm{~cm}^{-1},-\mathrm{CH}_{2}$ a $1451 \mathrm{~cm}^{-1}$, se identificó el modo vibracional del carbonilo, $\mathrm{CO}$ a $1737 \mathrm{~cm}^{-1}$ pero no se observó el -OH del ácido, por lo cal se trata de un ácido esterificado. A $1153 \mathrm{~cm}^{-1}$ se identificó el grupo éster, C-O; mientras que $\operatorname{los} \mathrm{C}=\mathrm{C}$ se identificaron a $1640 \mathrm{~cm}^{-}$ ${ }^{1}$ (banda de intensidad pequeña).

El espectro correspondiente al PCM/MSN se muestra en la Figura 3c, donde se observa el espectro de la sílice modificado con señales intensas correspondientes al PCM, los $\mathrm{CH}$ se observaron como un conjunto de señales anchas entre 2900 y $3000 \mathrm{~cm}^{-1}$, la señal del carbonilo se presentó a $1730 \mathrm{~cm}-1$ (señal ancha y de intensidad media) y los $-\mathrm{CH}_{2}$ a $1455 \mathrm{~cm}^{-1}$, estos se mostraron como señales de intensidad pequeña. (a)

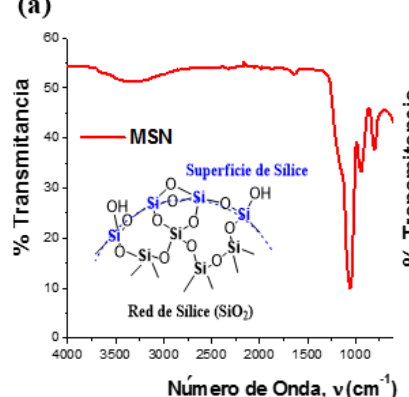

(b)

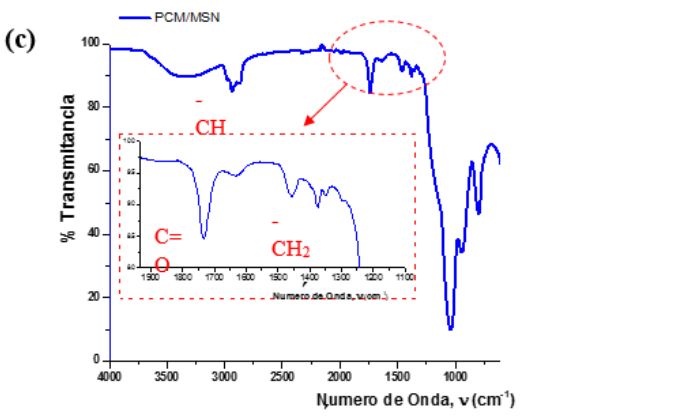

Figura 3 Espectros de infrarrojo (a) MSN (b) PCM (c) PCM adsorbido en la MSN

De acuerdo con los resultados de infrarrojo, el método de síntesis propuesto permite encapsular en la MSN el material de cambio de fase. Para determinar la cantidad de PCM adsorbido se realizó el análisis termogravimétrico (véase Figura 5). Donde la MSN mostró solo un tipo de caída de peso (pico 1) que se asignó a pérdida de disolvente; esta sílice tiene un rendimiento cerámico (porcentaje neto de red de sílice formada) del $84.43 \%$. La MSN/PCM mostraron tres diferentes caídas de peso; la primera corresponde a pérdida de disolvente (pico 1) y las otras dos a pérdida de materia orgánica (Picos 2 y 3). La cantidad de disolvente y materia orgánica presente en los materiales se calcularon por el método integral y son indicados en la Tabla 1.

\begin{tabular}{|l|c|c|c|}
\cline { 2 - 4 } \multicolumn{1}{c|}{} & \multicolumn{2}{l}{$\%$ en Peso } & \multicolumn{1}{l|}{} \\
$\mathrm{SiO}_{2}$ & Disolvente & $\begin{array}{l}\text { Materia } \\
\text { Orgánica }\end{array}$ \\
\hline MSN & 84.43 & 15.57 & --- \\
\hline MSN/PCM-1mL & 64.76 & 9.47 & 25.76 \\
\hline MSN/PCM-3mL & 62.78 & 9.89 & 27.32 \\
\hline
\end{tabular}

Tabla 1 Composición de MSN y MSN/PCM

Aunque la cantidad de PCM que se colocó para adsorber fue diferente, ambos materiales alcanzaron un valor de rendimiento cerámico similar, lo cual se refleja en una capacidad máxima de adsorción del PCM alrededor del $27 \%$ en peso. 


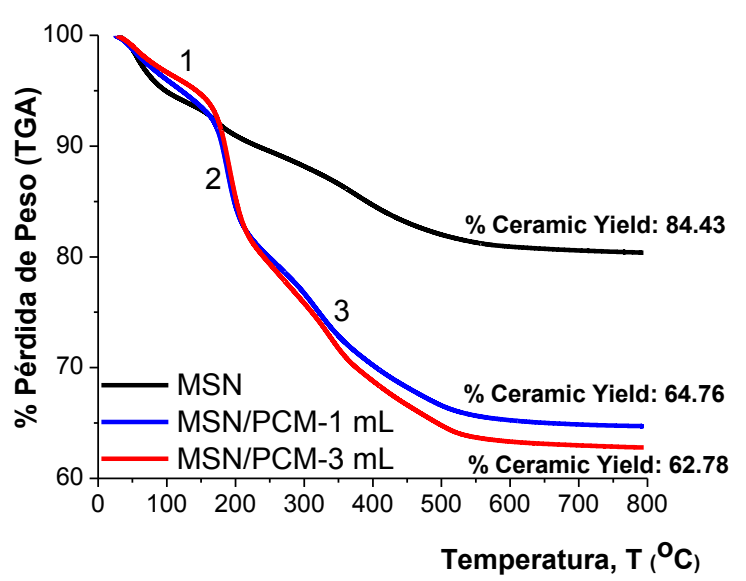

Figura 5 TGA para MSN y PCM encapsulado en la sílice

\section{Porosidad Formada para MSN y MSN/PCM}

La MSN es sintetizada por el método de Stöber, el cual promueve la hidrólisis y condensación del precursor de sílice (TEOS) bajo una catálisis alcalina que tiende a formar sílices no porosas. Lo anterior se observó con las isotermas de adsorción-desorción de $\mathrm{N}_{2}$ (Figura 6) obteniéndose para estos materiales una isoterma tipo III; donde la interacción del sorbato con la superficie es débil. La Tabla 2 resume las propiedades texturales de la sílice, donde se puede observar que el volumen de poro disminuye en $73.80 \%$; mientras que el área superficial de BET en $43 \%$.

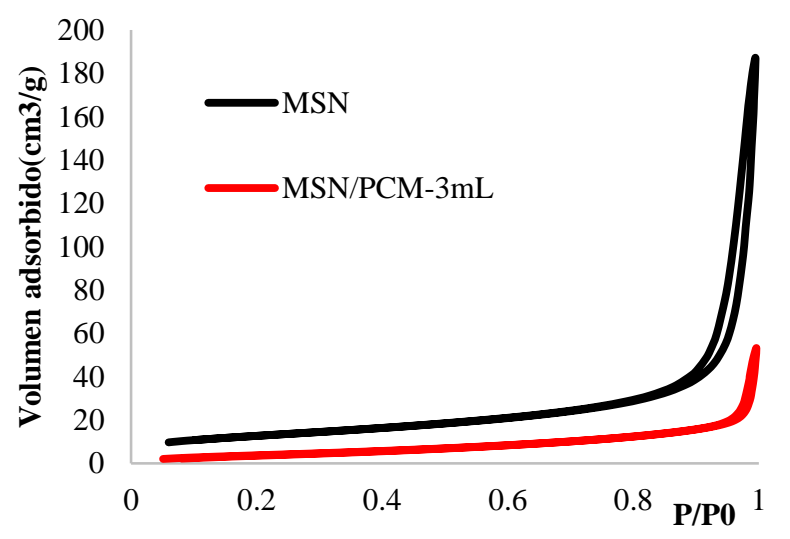

Figura 6 Isoterma de adsorción-desorción de $\mathrm{N}_{2}$

\begin{tabular}{|l|r|r|r|}
\cline { 2 - 4 } \multicolumn{1}{c|}{} & \multicolumn{1}{c}{$\begin{array}{c}\mathbf{A}_{\text {BET }} \\
\left(\mathbf{m}^{2} / \mathbf{g}\right)\end{array}$} & \multicolumn{1}{c|}{$\begin{array}{c}\mathbf{V}_{\text {poro }} \\
\left(\mathbf{c m}^{\mathbf{3}} \mathbf{g}\right)\end{array}$} & \multicolumn{1}{c|}{$\begin{array}{c}\mathbf{D}_{\text {poro }} \\
(\mathbf{n m})\end{array}$} \\
\hline MSN & 10.44 & 0.061576 & 6.24 \\
\hline MSN/PCM-3mL & 5.98 & 0.016129 & 5.56 \\
\hline
\end{tabular}

Tabla 2 Propiedades Texturales

\section{Caracterización Morfológica}

La Figura 7 muestra la micrografía observada para los materiales, la MSN son partículas esféricas monodispersas con tamaño promedio de $258.6 \mathrm{~nm}$.
Al adicionar el PCM $81 \mathrm{~mL}$ ) se observaron dos dispersiones de tamaño de partícula una de $2552 \mathrm{~nm}$ (tamaño original de la MSN) y otro de $107 \mathrm{~nm}$ (MSN/PCM). Para MSN/PCM-3mL se obtienen partículas esféricas monodispersas con tamaño promedio de $93 \mathrm{~nm}$.

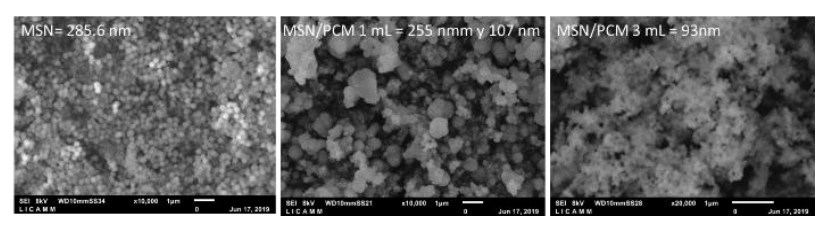

Figura 7 MEB de MSN y MSN con PCM adsorbido

\section{Caracterización Térmica}

La Figura 8 muestra el análisis diferencial calorimétrico, donde se puede identificar para la MSN una sola reacción endotérmica a los $100^{\circ} \mathrm{C}$, que se debe a la liberación de disolvente. Para la MSN/PCM se presentó esta reacción, además de 4 reacciones exotérmicas que ocurren a $30^{\circ} \mathrm{C}$ (1Еxo), $193^{\circ} \mathrm{C}$ (2Exo), $351^{\circ} \mathrm{C}$ (3Еxo) y $459^{\circ} \mathrm{C}$ (4Exo). Las reacciones de 2 a 3 Exo se deben a la descomposición del PCM; sin embargo, la reacción a $\operatorname{los} 30^{\circ} \mathrm{C}$ es un pico exotérmico de liberación de energía que es observado debido al almacenamiento de calor latente de conformación en la estructura orgánica del PCM. La Tabla 4 indica las cantidades de calor liberado por el PCM encapsulado en la MSN.

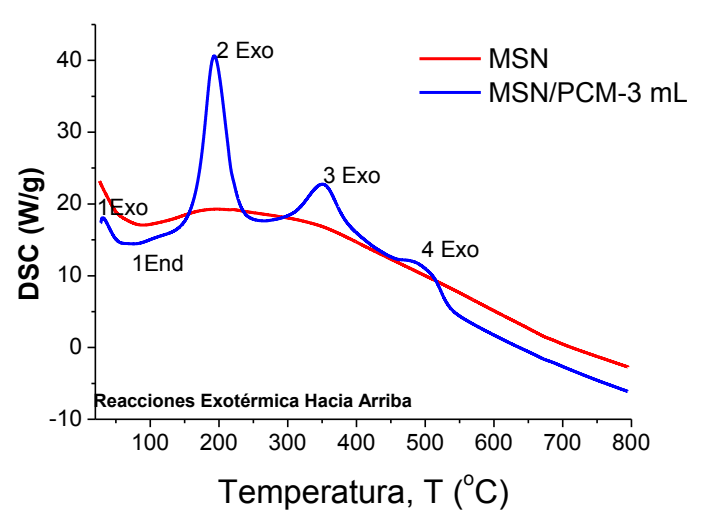

Figura 8 DSC para MSN y PCM encapsulado en la MSN

\begin{tabular}{|r|r|l|}
\hline Temperatura & \multicolumn{1}{c}{$\begin{array}{c}\text { Flujo de Calor } \\
\text { (W/g) }\end{array}$} & \multicolumn{1}{c|}{ Tipo de Calor } \\
\hline $30^{\circ} \mathrm{C}$ & 0.407 & $\begin{array}{l}\text { Almacenamiento } \\
\text { (reversible) }\end{array}$ \\
\hline $193^{\circ} \mathrm{C}$ & 2.555 & $\begin{array}{l}\text { Descomposición } \\
\text { (irreversible) }\end{array}$ \\
\hline $351^{\circ} \mathrm{C}$ & 1.731 & $\begin{array}{l}\text { Descomposición } \\
\text { (irreversible) }\end{array}$ \\
\hline $459^{\circ} \mathrm{C}$ & 0.646 & $\begin{array}{l}\text { Descomposición } \\
\text { (irreversible) }\end{array}$ \\
\hline
\end{tabular}

Tabla 4 Calor almacenado en MSN/PCM-3Ml

GONZÁLEZ-CERVANTES, Nancy, SALAZAR-HERNÁNDEZ, Mercedes, CANO-LARA, Miroslava y SALAZAR-HERNÁNDEZ, Carmen. Sílice Mesoporosa como encapsulador de materiales de cambio de fase (PCM). Revista de Ingeniería Tecnológica. 2019 


\section{Conclusiones}

El Pluronic F-127 es un surfactante neutro que permite encapsular aceite vegetal en MSN como se observó en la caracterización química y física. Sin embargo, existe un porcentaje máximo de adsorción del PCM que se limita al 27\%. En los MSN/PCM puede aprovecharse dos tipos de calor el reversible a $30^{\circ} \mathrm{C}(0.407 \mathrm{~W} / \mathrm{g})$ y el irreversible que es la reacción de descomposición del orgánico en la sílice que va entre $193-459{ }^{\circ} \mathrm{C}(4.93 \mathrm{~W} / \mathrm{g})$.

\section{Agradecimientos}

Los autores agradecen a la Secretaria de Investigación y Posgrado del Instituto Politécnico Nacional por el apoyo brindado a través del proyecto SIP-20195874. Además se agradece al LICAMM-UG por su apoyo en la caracterización por MEB.

\section{Referencias}

[1] Prim E.O, Thermal energy storage (TES) using phase change materials (PCM) for cold applications (2012) Phd Thesis. Lleida University. España

[2] Villasmil W, Fischer L.J, Worlitschek J, A review and evaluation of thermal insulation materials and methods for thermal energy storage systems, Renewable and Sustainable Energy Reviews (2019) 103, 71-4

[3] Zhou Xiangfa (2018) Porous Ceramic Matrix Phase Change Composites for Thermal Control Purposes of Hypersonic Vehicle. In Advances in some hypersonic vehicles technology. Pp. 153174. Intech Open Science. http://dx.doi.org/10.5772/intechopen.70863

[4] Motahar S, Nikkam N, Alemrajabi A.A, Khodabandeh R, Toprak m.S, Muhammed M, A novel phase change material containing mesoporous silica nanoparticles for thermal storage: A study on thermal conductivity and viscosity, International Communications in Heat and Mass Transfer (2014) 56, 114-120

[5] Qian T, Li J, Min X, Fan B, Integration of Pore Confinement and Hydrogen-Bond Influence on the Crystallization Behavior of C18 PCMs in Mesoporous Silica for Form-Stable Phase Change Materials, ACS Sustainable Chemistry \& Engineering (2018) 6, 897-908
[6] Peng S, Huang J, Wang T, Zhu P, Effect of fumed silica additive on supercooling, thermal reliability and thermal stability of $\mathrm{Na}_{2} \mathrm{HPO}_{4} \cdot 12 \mathrm{H}_{2} \mathrm{O}$ as inorganic PCM, Thermochimica Acta (2019) 675, 1-8

[7] Kohno Y, Haga E, Yoda K, Shibita M, Fukuhara Ch, Tomita Y, Maeda Y, Kobayashi $\mathrm{K}$, Adsorption behavior of natural anthocyanin dye on mesoporous silica, Journal of physics and chemistry of solids (2014) 75, 48-51

[8] Burke A, Hanrahan J.P, Healy D.A, Sodeau J.R, Holmes J.D, Morris M.A, Large pore bifunctionalised mesoporous silica for metal ion pollution treatment, Journal of hazardous materials (2009) 164, 229-234

[9] Li J, Miao X, Hao Y, Zhao J, Sun X, Wang L, Synthesis, amino-functionalization of mesoporous silica and its adsorption of $\mathrm{Cr}(\mathrm{VI})$, Journal of colloid and interface science (2008) 318, 309-314

[10] Wang Y, Xia T.D, Zheng H, Feng H.X, Stearic acid/silica fume composite as formstable phase change material for thermal energy storage, Energyand Buildings (2011) 43, 23652370

[11] Fang G, Li H, Liu X, Preparation and properties of lauric acid/silicon dioxide composites as form-stable phase change materials for thermal energy storage, Materials chemistry and physics (2010) 122, 533-536

[12] Liu S, ma G, Xie S, Jia Y, Sun J, Jing Y, Diverting the phase transition behaviour of adipic acid via mesoporous silica confinement, RSC Adv, 2016, 6, 111787-111796

[13] Launer P.J (1987) Infrared analysis of organosilicon compounds: spectra structure correlation silicon compounds. In: Arkles B (ed) Gelest Inc: Morrisville, PA 